\title{
INFLUENCE OF VARIOUS SOURCES OF ORGANIC NITROGEN AS A PARTIAL SUBSTITUTE FOR MINERAL NITROGEN FERTILIZER ON YIELD, FRUIT QUALITY AND LEAF MINERAL CONTENTS OF ZEBDA MANGO TREES
}

(Received: 20.11.2011)

\author{
By \\ R.I.Saad \\ Department of Tropical and Sub Tropical Fruits, Horticulture Research Institute, \\ Agricultural Research Center, Giza, Egypt.
}

\begin{abstract}
This investigation was conducted during three seasons (2008/2009, 2009/2010 and 2010/2011) on Zebda mango trees grown in sandy soil under flood irrigation condition. The recommended rate of nitrogen namely $700 \mathrm{gms} \mathrm{N} /$ tree/year was added either, completely via mineral source (ammonium sulphate $20.6 \%$ ) or at various ratios from organic fertilizer (town refuse and cattle manure). The study focused on the effect of these treatments on yield, physical and chemical properties of fruits and leaf mineral contents, to determine the maximum substitution proportion of organic nitrogen source (with best yield and fruit quality) instead of mineral nitrogen fertilizer.

The obtained results showed that all the used treatments of various sources of organic nitrogen were effective in improving yield, fruit quality and leaf mineral contents in comparison with mineral N 100\% (control). It was also noticed that the positive effect of the superior treatments on yield/tree, fruit weight and diameter, seed/pulp ratio, TSS, TSS/acid ratio, total sugars and fruit carotinoids could be arranged in the descending order with significant differences; mineral N 20\% + cattle manure 80\%; mineral N 50\% + town refuse $50 \%$ and mineral N 80\% + cattle manure 20\%. Moreover, both of mineral N 20\% + cattle manure $80 \%$ or mineral $\mathrm{N} \mathrm{50 \%} \mathrm{+} \mathrm{town} \mathrm{refuse} 50 \%$ significantly increased reducing sugars of fruits and leaf $\mathrm{P}, \mathrm{Mn}$ and $\mathrm{Zn}$ than those of other treatments.

Hence, it could be concluded that under the condition of this study, using mineral N $20 \%+$ cattle manure $80 \%$ is very useful for minimizing the use of mineral $\mathrm{N}$ fertilizer, reducing environmental pollution and obtaining an economical yield with high quality of Zebda mango fruits and considered a good recommendation for fertilizing mango trees under resembling conditions.
\end{abstract}

Key words: fruit quality, leaf mineral content, mango trees, mineral fertilizer, organic fertilizer, yield.

\section{INTRODUCTION}

Mango trees are considered one of the main fruits in Egypt. It ranks the fourth after citrus, grapes and olives. The total acreage of mango in Egypt reached 184204 feddans, producing about 532422 tons (Ministry of Agric., ARE,2008).

Fertilization is the most important factor which affect mango yield and fruit quality. Growers add high quantaties of chemical fertilizers to increase the yield of their trees, but the efficiency of nitrogen fertilizers practically under field conditions and surface irrigation rarely exceeds $50 \%$ and it is usually only $30-40 \%$ (Englestad and Russel, 1975 and Sahrawat, 1979).

Such low efficiency may be due to the losses of $\mathrm{N}$ by leaching of nitrate, or reduction of nitrate resulting in the formation of nitrogen gas that is lost by volatilization (Goring, 1962). Leaching of nitrate and down word movement below the rooting zone with flowing water causes many problems such as nitrate pollution of ground water and growing crops (Gambrell et al., 1975).

Nowadays, great attention has been focused on the possibility of using organic fertilizers for enhancing growth and fruit quality, consequently depress the pollution occurred in the Egyptian environment due to the exaggeration in the application of chemical fertilizers.

Organic fertilization has many advantages, it improves soil structure and reduces soil $\mathrm{pH}$, results in better root growth and more efficient use of water and nutrients. It also provides slow release of nitrogen and other nutrients. In addition, organic fertilization increases the nutrient-holding properties of soil which lead to the lowering the need for excessive applications of nitrogen 
fertilizer, hence decrease nitrate contamination of ground water (El-Sayed, 1994; Casale et al., 1995; Ahmed et al., 1996; El-Kassas et al., 1997; Nassar, 1998 and Harhash \& Abd El-Nesser, 2000).

The results of many studies reviewed the
The selected trees were treated by the recommended rate of actual nitrogen i.e., 700 gms/tree/year from different amounts of the studied sources (according to their chemical analysis (Table 2) represented in the following treatments :

\begin{tabular}{|l|c|c|c|}
\hline \multicolumn{1}{|c|}{ Treatments } & \multicolumn{3}{|c|}{ Amounts of fertilizer (Kg/tree/year) } \\
\cline { 2 - 4 } & $\mathbf{2 0 0 8 / 2 0 0 9}$ & $\mathbf{2 0 0 9 / 2 0 1 0}$ & $\mathbf{2 0 1 0 / 2 0 1 1}$ \\
\hline 1- Mineral nitrogen only "MN" 100\% (control). & 3.4 & 3.4 & 3.4 \\
2- Mineral nitrogen "MN" 80\%+Town refuse "TR" 20\%. & $2.7+16.3$ & $2.7+15.1$ & $2.7+15.9$ \\
3- Mineral nitrogen "MN" 50\%+Town refuse "TR" 50\%. & $1.7+40.7$ & $1.7+37.6$ & $1.7+39.8$ \\
4- Mineral nitrogen "MN" 20\%+Town refuse "TR" 80\%. & $0.7+65.1$ & $0.7+60.2$ & $0.7+63.6$ \\
5- Mineral nitrogen "MN" 80\%+Cattle manure "CM" 20\%. & $2.7+12.4$ & $2.7+12.7$ & $2.7+12.8$ \\
6- Mineral nitrogen "MN" 50\%+Cattle manure "CM" 50\%. & $1.7+31.0$ & $1.7+31.8$ & $1.7+32.1$ \\
7- Mineral nitrogen "MN" 20\%+Cattle manure "CM" 80\%. & $0.7+49.6$ & $0.7+50.9$ & $0.7+51.3$ \\
\hline
\end{tabular}

importance of organic manures for stimulating growth and productivity of some fruit crops ; Smith, 1998; Joo et al., 1999; Tiwary et al., 1999; Abd El-Naby \& Gomaa, 2000; Geetha \& Nair, 2000; Shahein et al., 2003; Chatzitheodorou et al., 2004; El-Assar, 2005; Omar \& Belal, 2007; AlWasfy \& El-Khawaga, 2008; Abd El-Salam et al., 2009 and Shaheen et al., 2009.

In addition, the application of organic $\mathrm{N}$ sources with a mineral $\mathrm{N}$ was accompanied with an obvious promotion on fruit quality of many cultivars of fruit trees (Gouda, 1984; Salem, 1986; Cole et al., 1987; Dahdoh \& El- Hassanin, 1993; Li et al., 1998; Abd El-Nasser \& Harhash, 2000; Abd El-Hameed \& Ragab, 2004; Mansour et al., 2004; Mohamed \& Gobara, 2004; Diab, 2006; Abd El-Rahman, 2007; Saad \& Saad, 2007 and Hossam El-Deen \& Boshra, 2008).

Therefore, this study aimed to examine the beneficial effect of two forms of organic nitrogen sources namely; town refuse and cattle manure in combination with a mineral nitrogen source on yield, fruit quality and leaf mineral contents of Zebda mango trees.

\section{MATERIALS AND METHODS}

This study was carried out in a private orchard situated at Edko region, El-Behira Governorate, during the three successive seasons of 2008/2009, 2009/2010 and 2010/2011 on Zebda mango trees. All trees were 10 year old and grafted on a seedling rootstalk, grown in sandy soil and planted at $5 \times 5$ meters apart. Soil analysis was carried out and listed in Table (1).

Twenty eight trees, as uniform as possible were chosen. Regular agricultural practices such as hoeing, irrigation and pest management were done as usual to all the studied trees.
The treatments were arranged in Randomized Complete Blocks Design, in which each treatment was replicated four times, with one tree for each replicate; i.e., 7 treatments $\times 4$ replicates $=28$ trees in total.

The amounts of mineral nitrogen source (ammonium sulphate $20.6 \% \mathrm{~N}$ ) were applied broadcasted at 3 equal batches in February, May and July. However, the amounts of organic nitrogen sources (town refuse and cattle manure) were mixed with the surface of the soil and applied once under the tree canopy in trenches (50 $\mathrm{cm}$ width $\times 50 \mathrm{~cm}$ depth) arround the tree in the beginning of December of each season. Town refuse fertilizer used was produced by Abees factory for organic fertilizers (belong to Alex. Governorate). In addition, each experimental tree received $1.5 \mathrm{kgms} /$ year of superphosphate $(45.5 \%$ $\left.\mathrm{P}_{2} \mathrm{O}_{2}\right)$ in December and $1.25 \mathrm{kgms} / \mathrm{year}$ of potassium sulphate $\left(50 \% \mathrm{~K}_{2} \mathrm{O}_{2}\right)$ at 2 equal batches in April and June in each season.

The following topics were studied :

1- Flowering indices : At full bloom (at the end of April), the number of panicles per tree and the number of flowers per panicle were counted (32 panicles from each replicate were randomly chosen and labeled for this purpose).

- 2-Yield indices : After fruit set (in the mid of May), the number of fruit set at initial fruit set and at harvest were recorded. Fruit set percentage was calculated as the following equation:

$$
\text { Fruit } \text { set } \%=\frac{\text { No. of fruits set / panicle }}{\text { Total No. of flowers / panicle }}
$$


Table (1): Chemical and physical characteristics of the experimental soil.

\begin{tabular}{|c|c|c|c|}
\hline Character & Value & Character & Value \\
\hline $\mathrm{EC}\left(\mathrm{dsm}^{-1}\right)$ & 1.53 & Soluble anions $(\mathrm{meq} / \mathrm{L})$ & \\
\hline $\mathrm{pH}(1: 2.5)$ & 8.11 & $\mathrm{SO}_{+}^{2-}$ & 10.37 \\
\hline $\mathrm{CaCO}_{3}(\%)$ & 6.32 & & 5.98 \\
\hline Organic matter $(\%)$ & 0.21 & $\mathrm{Cl}^{-}$ & 7.45 \\
\hline Total N (\%) & 0.03 & $\mathrm{HCO}_{3}^{-}$ & \\
\hline Soluble cations $(\mathrm{meq} / \mathrm{L})$ & & Particle size distribution (\%) & \\
\hline $\mathrm{Ca}^{2+}$ & 7.10 & Sand & 82.46 \\
\hline $\mathrm{Mg}^{2+}$ & 3.39 & Silt & 2.51 \\
\hline $\mathrm{Na}^{+}$ & 13.20 & Clay & 15.03 \\
\hline $\mathrm{K}^{+}$ & 0.08 & Texture class & Sandy \\
\hline
\end{tabular}

Table(2): Chemical analysis of the tested organic fertilizers.

\begin{tabular}{|c|c|c|c|c|c|c|}
\hline \multirow{2}{*}{ Constituents } & \multicolumn{3}{|c|}{ Town refuse $(\mathrm{TR})$} & \multicolumn{3}{|c|}{ Cattle manure (CM) } \\
\hline & $2008 / 2009$ & $2009 / 2010$ & $2010 / 2011$ & $2008 / 2009$ & $2009 / 2010$ & $2010 / 2011$ \\
\hline$\%$ & & & & & & \\
\hline Moisture content & 5.5 & 5.2 & 4.8 & 19.3 & 17.6 & 18.2 \\
\hline Organic matter & 29.8 & 25.17 & 24.12 & 20.55 & 22.16 & 21.48 \\
\hline Organic carbon & 21.53 & 20.91 & 18.10 & 17.51 & 18.14 & 19.05 \\
\hline Total N & 0.86 & 0.93 & 0.88 & 1.13 & 1.10 & 1.09 \\
\hline $\mathrm{P}$ & 0.29 & 0.31 & 0.36 & 0.71 & 0.59 & 0.67 \\
\hline K & 1.34 & 1.36 & 1.25 & 1.15 & 1.30 & 1.19 \\
\hline $\mathrm{Ca}$ & 4.33 & 4.07 & 3.91 & 1.37 & 1.28 & 1.45 \\
\hline $\mathrm{Mg}$ & 0.29 & 0.31 & 0.20 & 0.69 & 0.73 & 0.82 \\
\hline $\mathrm{C} / \mathrm{N}$ ratio & $25: 1$ & $22: 1$ & $21: 1$ & $16: 1$ & $16: 1$ & $17: 1$ \\
\hline ppm & & & & & & \\
\hline $\mathrm{Fe}$ & 513 & 396 & 401 & 149 & 151 & 158 \\
\hline $\mathrm{Zn}$ & 580 & 677 & 562 & 125 & 90 & 131 \\
\hline $\mathrm{Mn}$ & 401 & 329 & 388 & 74 & 89 & 120 \\
\hline $\mathrm{Cu}$ & 205 & 310 & 276 & 38 & 19 & 26 \\
\hline
\end{tabular}

Yield was calculated as the weight of fruit $x$ number of fruits per tree at harvest time (mid September).

\section{3- Fruit physical and chemical properties}

Samples of 16 fruits from each replicate (tree) were used to determine the physical and chemical properties. In this respect, fruit dimentions and weight of the fruit, pulp, seed and peel were recorded, also seed/pulp ratio was calculated. Chemical properties, included total soluble solid percentage (T.S.S.\%) was determined by a hand refractometer, total acidity percentage (expressed as gm citric acid/100 gm pulp) (A.O.A.C., 1995). TSS/acid ratio was calculated, total and reducing sugars and caroteniods were determined according to Malik \& Singh (1980) and Roy (1973), respectively.

4- Leaf mineral content Samples comprised of twenty mature leaves were collected " mid July of each season" from the middle of vegetative shoots around each of the chosen tree. For mineral content determination, nitrogen $(\mathrm{N})$ and phosphorus $(\mathrm{P})$ were determined according to venhuis (1976) and Murphy \& Riley (1962), respectively. Potassium (K) was determined by Flame photometer, iron $(\mathrm{Fe})$, manganese $(\mathrm{Mn})$ and zinc $(\mathrm{Zn})$ were determined by Perkin Elmer Atomic Absorption Spectrophotometer. The obtained data were statistically analyzed according to Mead et al. (1993) using New L.S.D test for examining the significant differences between the studied treatments.

\section{RESULTS AND DISCUSSION}

\subsection{Effect of different organic nitrogen sources on yield components}

Data presented in Table (3) revealed that yield components of Zebda mango trees namely; number of panicle/tree, number of flowers/panicle, number of set fruits/panicle at initial fruit set, fruit set percentage, number of fruits/tree and yield/tree were improved by different organic nitrogen sources as compared with mineral nitrogen only. The obtained results showed that the treatment of MN 20\%+CM $80 \%$ significantly increased the number of panicle/tree, the number of set fruits at 
Table (3): Effect of different organic nitrogen sources on yield components of Zebda mango trees in 2008/2009, 2009/2010 and 2010/2011 seasons.

\begin{tabular}{|c|c|c|c|c|c|c|c|c|c|}
\hline \multirow[t]{2}{*}{ Treatments } & \multicolumn{3}{|c|}{ No. of panicles/tree } & \multicolumn{3}{|c|}{ No. of flowers/panicle } & \multicolumn{3}{|c|}{$\begin{array}{l}\text { No. of set fruits/panicle at initial } \\
\text { fruit set }\end{array}$} \\
\hline & 2008/2009 & $2009 / 2010$ & $2010 / 2011$ & $2008 / 2009$ & $2009 / 2010$ & $2010 / 2011$ & $2008 / 2009$ & $2009 / 2010$ & $2010 / 2011$ \\
\hline 1-Mineral nitrogen "MN" 100\% (control) & $67.69^{\mathrm{c}}$ & $35.55^{\mathrm{d}}$ & $64.67^{\mathrm{d}}$ & 963.7 & $791.3^{\mathrm{b}}$ & $975.0^{\mathrm{c}}$ & $154.0^{\mathrm{e}}$ & $132.1^{\mathrm{de}}$ & $158.4^{\mathrm{d}}$ \\
\hline 2- Mineral nitrogen "MN" 80\%+ Town refuse "TR" 20\% & $71.27^{\mathrm{ab}}$ & $43.11^{\mathrm{c}}$ & $68.72^{\mathrm{cd}}$ & 990.3 & $817.0^{\mathrm{ab}}$ & $991.5^{\mathrm{abc}}$ & $172.8^{\mathrm{d}}$ & $148.8^{\mathrm{c}}$ & $171.4^{\mathrm{c}}$ \\
\hline 3- Mineral nitrogen "MN" 50\%+ Town refuse "TR" 50\% & $78.02^{\mathrm{a}}$ & $48.36^{\mathrm{a}}$ & $77.37^{\mathrm{a}}$ & 994.5 & $829.8^{\mathrm{ab}}$ & $1005.6^{\mathrm{a}}$ & $201.5^{\mathrm{b}}$ & $168.0^{\mathrm{ab}}$ & $204.1^{\mathrm{a}}$ \\
\hline 4- Mineral nitrogen "MN" 20\%+ Town refuse "TR" $80 \%$ & $73.50^{\mathrm{b}}$ & $45.47^{\mathrm{bc}}$ & $72.86^{\mathrm{bc}}$ & 991.7 & $822.2^{\mathrm{ab}}$ & $1000.9^{\mathrm{a}}$ & $189.1^{\mathrm{c}}$ & $159.4^{\mathrm{b}}$ & $184.7^{\mathrm{b}}$ \\
\hline 5- Mineral nitrogen "MN" 80\%+ Cattle manure "CM" 20\% & $67.98^{\mathrm{c}}$ & $44.24^{\mathrm{c}}$ & $71.95^{\mathrm{bc}}$ & 987.2 & $826.0^{\mathrm{a}}$ & $997.9^{\mathrm{ab}}$ & $177.7^{\mathrm{d}}$ & $141.6^{\mathrm{cd}}$ & $180.3^{\mathrm{b}}$ \\
\hline 6- Mineral nitrogen "MN" 50\%+ Cattle manure "CM" 50\% & $72.61^{\mathrm{b}}$ & $43.81^{\mathrm{c}}$ & $70.59^{\mathrm{c}}$ & 994.0 & $814.6^{\mathrm{ab}}$ & $978.3^{\mathrm{bc}}$ & $156.4^{\mathrm{e}}$ & $168.0^{\mathrm{ab}}$ & $161.1^{\mathrm{d}}$ \\
\hline 7- Mineral nitrogen "MN" 20\%+ Cattle manure "CM" 80\% & $79.23^{\mathrm{a}}$ & $48.81^{\mathrm{a}}$ & $79.60^{\mathrm{a}}$ & 999.7 & $843.7^{\mathrm{a}}$ & $1010.3^{\mathrm{a}}$ & $209.9^{\mathrm{a}}$ & $176.3^{\mathrm{a}}$ & $205.6^{\mathrm{a}}$ \\
\hline New L.S.D. at 5\% & 4.53 & 2.88 & 4.50 & N.S. & 39.5 & 21.5 & 5.1 & 9.8 & 6.6 \\
\hline
\end{tabular}

Cont.Table (3): Effect of different organic nitrogen sources on yield components of Zebda mango trees in 2008/2009, 2009/2010 and 2010/2011 seasons.

\begin{tabular}{|c|c|c|c|c|c|c|c|c|c|}
\hline \multirow{2}{*}{ Treatments } & \multicolumn{3}{|c|}{ Fruit set \% } & \multicolumn{3}{|c|}{ No. of fruits/tree } & \multicolumn{3}{|c|}{ Yield/tree $(\mathrm{Kg})$} \\
\hline & $2008 / 2009$ & $2009 / 2010$ & $2010 / 2011$ & $2008 / 2009$ & $2009 / 2010$ & $2010 / 2011$ & $2008 / 2009$ & $2009 / 2010$ & $2010 / 2011$ \\
\hline 1-Mineral nitrogen "MN" 100\% (control) & $15.99^{\mathrm{d}}$ & $16.70^{\mathrm{d}}$ & $16.28^{\mathrm{cd}}$ & $49.68^{\mathrm{e}}$ & $18.78^{\mathrm{f}}$ & $54.26^{\mathrm{e}}$ & $20.83^{\mathrm{g}}$ & $7.90^{\mathrm{g}}$ & $22.74^{\mathrm{g}}$ \\
\hline 2- Mineral nitrogen "MN" 80\%+ Town refuse "TR" $20 \%$ & $18.42^{\mathrm{c}}$ & $18.26^{\mathrm{bc}}$ & $17.17^{\mathrm{c}}$ & $53.47^{\mathrm{d}}$ & $22.64^{\mathrm{e}}$ & $56.20^{\mathrm{e}}$ & $23.13^{\mathrm{f}}$ & $9.93^{\mathrm{f}}$ & $24.31^{\mathrm{f}}$ \\
\hline 3- Mineral nitrogen "MN" 50\%+ Town refuse "TR" 50\% & $20.27^{\mathrm{ab}}$ & $20.63^{\mathrm{a}}$ & $20.87^{\mathrm{a}}$ & $75.13^{\mathrm{a}}$ & $43.48^{\mathrm{a}}$ & $76.45^{\mathrm{b}}$ & $35.66^{\mathrm{b}}$ & $21.00^{\mathrm{b}}$ & $36.31^{\mathrm{b}}$ \\
\hline 4- Mineral nitrogen "MN" 20\%+ Town refuse "TR" $80 \%$ & $19.03^{\mathrm{bc}}$ & $19.31^{\mathrm{b}}$ & $18.32^{\mathrm{b}}$ & $70.48^{\mathrm{b}}$ & $39.18^{\mathrm{c}}$ & $71.40^{\mathrm{c}}$ & $31.86^{\mathrm{d}}$ & $17.90^{\mathrm{d}}$ & $32.28^{\mathrm{d}}$ \\
\hline 5- Mineral nitrogen "MN" 80\%+ Cattle manure "CM" 20\% & $18.00^{\mathrm{c}}$ & $17.47^{\mathrm{cd}}$ & $18.22^{\mathrm{b}}$ & $71.71^{\mathrm{b}}$ & $41.15^{\mathrm{b}}$ & $73.65^{\mathrm{c}}$ & $33.90^{\mathrm{c}}$ & $19.36^{\mathrm{c}}$ & $34.39^{\mathrm{c}}$ \\
\hline 6- Mineral nitrogen "MN" 50\%+ Cattle manure "CM" 50\% & $15.79^{\mathrm{d}}$ & $15.29^{\mathrm{e}}$ & $16.04^{\mathrm{d}}$ & $62.07^{\mathrm{c}}$ & $32.87^{\mathrm{d}}$ & $64.27^{\mathrm{d}}$ & $26.43^{\mathrm{e}}$ & $14.81^{\mathrm{e}}$ & $29.22^{\mathrm{e}}$ \\
\hline 7- Mineral nitrogen "MN" 20\%+ Cattle manure "CM" 80\% & $21.00^{\mathrm{a}}$ & $21.44^{\mathrm{a}}$ & $20.99^{\mathrm{a}}$ & $76.88^{\mathrm{a}}$ & $44.96^{\mathrm{a}}$ & $79.87^{\mathrm{a}}$ & $36.76^{\mathrm{a}}$ & $21.80^{\mathrm{a}}$ & $38.18^{\mathrm{a}}$ \\
\hline New L.S.D. at $5 \%$ & 1.30 & 1.19 & 1.00 & 2.23 & 1.83 & 2.38 & 1.06 & 0.97 & 1.02 \\
\hline
\end{tabular}

The values per column followed by the same letter do not differ at $5 \%$ level of significance.

$\mathrm{MN}=$ Mineral nitrogen ; $\mathrm{TR}=$ Town refuse $; \mathrm{CM}=$ Cattle manure. 
initial fruit set, fruit set percentage and the number of fruits/tree than those of the control and other treatments, except those of the treatment (MN 50\%+TR 50\%) which ranked the second effective treatment. In meantime, the promising treatment of $\mathrm{MN} 20 \%+\mathrm{CM} 80 \%$ significantly gave the highest yield/tree than those of all treatments. On the other hand, treatment of MN $100 \%$ "control" significantly gave the lowest values for all yield components, meanwhile the other treatments revealed an intermediate values of the studied yield components.

The positive effect of organic manures plus mineral nitrogen fertilizers on yield components i.e., no. of panicle/tree; no. of flowers/panicle; no. of set fruits/panicle, fruit set \%; no. of fruit/tree and yield/tree could be attributed to its role in increasing amino acid contents, which are considered as constituents of protein and other compounds that share in the development of new tissues (Smith, 1998 and Tiwary et al., 1999). Additionally, this pronounced effect of organic manures plus mineral $\mathrm{N}$ fertilizers on yield components may be due to an increase in cell division and enlargement and consequently increase in vegetative growth which is reflected on increasing yield components as a final result of the physiological processes (Abd El-Naby\& Gomma, 2000; Geetha \& Nair, 2000 and Shaheen et al., 2009). The obtained results are in harmony with those obtained by Omar \& Belal (2007) on mango trees and Shahein et al. (2003); El-Assar (2005); Al-Wasfy \& El-Khawaga (2008) on date palms.

3.2. Effect of different organic nitrogen sources on physical properties of Zebda mango fruits :

Data presented in Table (4) clearly showed that the two organic nitrogen sources used plus mineral nitrogen fertilizer significantly affected the physical properties of fruits. The maximum values of fruit weight, fruit length, fruit diameter and pulp weight were recorded for trees received (MN 20\%+ CM 80\%) treatment in most cases. Such treatment also significantly produced the lowest values of seed weight and seed/pulp ratio. These results were true in the three experimental seasons.

The promotion effect of organic nitrogen source (cattle manure) plus mineral nitrogen fertilizer on fruit weight, fruit length, fruit diameter and pulp weight could be attributed to its role on the availability of elements as constituents of protein and other compounds which produce the new tissues through their role as constituents of the nucleic acids DNA and RNA, in addition to the role of organic manure on increase the soil content of IAA and cytokines which stimulated the plant growth (Li et al., 1998). These results are in line with those of Geetha \& Nair (2000); Chatzitheodorou et al. (2004); Abd El-Rahman (2007); Saad \& Saad (2007) and Hossam \& Boshra (2008).

\subsection{Effect of different organic nitrogen sources on the chemical characteristics of Zebda mango fruits}

Data presented in Table (5) showed that the application of the studied organic nitrogen sources plus mineral nitrogen fertilizers significantly improved the chemical characteristics of Zebda mango fruits in terms of total soluble solids (TSS\%), TSS/acid ratio, reducing \& total sugars and carotinoids as compared with the application of the mineral nitrogen source. The present treatments could be arranged according to their significant effect on such chemical parameters in the following descending order; treatment of (MN $20 \%+\mathrm{CM} 80 \%)$ and (MN 50\%+TR 50\%), (MN $80 \%+\mathrm{CM} 20 \%),(\mathrm{MN} 20 \%+\mathrm{TR} 80 \%)$, (MN $80 \%+$ TR 20\%), (MN 50\% + CM 50\%) and (MN 100\%, control). In meantime, there was no significant difference between the effect of (MN $20 \%+\mathrm{CM} 80 \%$ ) treatment (MN 50\%+TR 50\%) on reducing sugars. However, treatment (MN $100 \%$, control) gave significantly the highest total acidity content of fruit jouice as compared with the other treatments.These obtained results were true in the three seasons.

Such findings might be due to the effect of organic nitrogen sources which ascribed a good balance between growth and fruiting resulting in accumulation of more carbohydrates and make them much available for enhancing chemical constituents of fruits.

The above mentioned results are in accordance with those obtained by Joo et al. (1999); Abd ElHameed \& Ragab (2004); Mohamed \& Gobara (2004); Mansour et al. (2004); Abd El-Salam et al. (2009) on different fruit crops.Diab (2006) indicated that increasing the organic nitrogen level substantially from $12.5 \%$ to $75 \%$ of recommended nitrogen improved the fruit quality of sewy date palms.

\subsection{Effect of different organic nitrogen sources on leaf mineral content of Zebda mango trees :}

The data in Table (6) clearly indicated that amending Zebda mango trees with the two organic nitrogen sources namely town refuse (TR) and cattle manure $(\mathrm{CM})$ combined with mineral 
Table (4): Effect of different organic nitrogen sources on physical properties of Zebda mango fruits in 2008/2009, 2009/2010 and 2010/2011 seasons.

\begin{tabular}{|c|c|c|c|c|c|c|c|c|c|}
\hline \multirow{2}{*}{ Treatments } & \multicolumn{3}{|c|}{ Fruit weight (gms) } & \multicolumn{3}{|c|}{ Fruit length $(\mathbf{c m})$} & \multicolumn{3}{|c|}{ Fruit diameter (cm) } \\
\hline & $2008 / 2009$ & $2009 / 2010$ & $2010 / 2011$ & $2008 / 2009$ & $2009 / 2010$ & 2010/2011 & $2008 / 2009$ & $2009 / 2010$ & 2010/2011 \\
\hline 1-Mineral nitrogen "MN" 100\% (control) & $419.0^{\mathrm{g}}$ & $421.9 \mathrm{~g}$ & $419.1 \mathrm{~g}$ & $12.333^{\mathrm{e}}$ & $12.036^{\mathrm{g}}$ & $12.063^{\mathrm{g}}$ & $7.320^{\mathrm{g}}$ & $7.273 \mathrm{~g}$ & $7.346^{\mathrm{g}}$ \\
\hline 2- Mineral nitrogen "MN" 80\%+ Town refuse "TR" 20\% & $432.6^{\mathrm{e}}$ & $438.6^{\mathrm{f}}$ & $432.6^{\mathrm{f}}$ & $13.983^{\mathrm{d}}$ & $14.031^{\mathrm{f}}$ & $13.913^{\mathrm{f}}$ & $7.520^{\mathrm{f}}$ & $7.672^{f}$ & $7.643^{f}$ \\
\hline 3- Mineral nitrogen "MN" 50\%+ Town refuse "TR" 50\% & $474.6^{\mathrm{b}}$ & $482.9^{\mathrm{b}}$ & $475.0^{\mathrm{b}}$ & $14.683^{\mathrm{a}}$ & $14.691^{\mathrm{b}}$ & $14.803^{\mathrm{b}}$ & $7.670^{\mathrm{b}}$ & $7.873^{\mathrm{b}}$ & $8.130^{\mathrm{b}}$ \\
\hline 4- Mineral nitrogen "MN" 20\%+ Town refuse "TR" 80\% & $452.0^{\mathrm{d}}$ & $456.8^{\mathrm{d}}$ & $452.1^{\mathrm{e}}$ & $14.223^{\mathrm{c}}$ & $14.235^{\mathrm{d}}$ & $14.273^{\mathrm{d}}$ & $7.580^{\mathrm{d}}$ & $7.753^{\mathrm{d}}$ & $7.713^{\mathrm{d}}$ \\
\hline 5- Mineral nitrogen "MN" 80\%+ Cattle manure "CM" 20\% & $472.7^{\mathrm{c}}$ & $470.5^{\mathrm{c}}$ & $466.9^{\mathrm{c}}$ & $14.453^{\mathrm{b}}$ & $14.470^{\mathrm{c}}$ & $14.423^{\mathrm{c}}$ & $7.630^{\mathrm{c}}$ & $7.821^{\mathrm{c}}$ & $7.735^{\mathrm{c}}$ \\
\hline 6- Mineral nitrogen "MN" 50\%+ Cattle manure "CM" 50\% & $425.8^{\mathrm{f}}$ & $450.6^{\mathrm{e}}$ & $654.6^{\mathrm{d}}$ & $14.203^{\mathrm{c}}$ & $14.205^{\mathrm{e}}$ & $14.233^{\mathrm{e}}$ & $7.560^{\mathrm{e}}$ & $7.730^{\mathrm{e}}$ & $7.670^{\mathrm{e}}$ \\
\hline 7- Mineral nitrogen "MN" 20\%+ Cattle manure "CM" 80\% & $477.9^{\mathrm{a}}$ & $484.9^{\mathrm{a}}$ & $478.1^{\mathrm{a}}$ & $14.693^{\mathrm{a}}$ & $14.869^{\mathrm{a}}$ & $14.993^{\mathrm{a}}$ & $7.690^{\mathrm{a}}$ & $7.886^{\mathrm{a}}$ & $8.183^{\mathrm{a}}$ \\
\hline New L.S.D. at $5 \%$ & 1.4 & 1.2 & 1.5 & 0.176 & 0.174 & 0.181 & 0.019 & 0.012 & 0.017 \\
\hline
\end{tabular}

Cont. Table (4): Effect of different organic nitrogen sources on physical properties of Zebda mango fruits in 2008/2009, 2009/2010 and 2010/2011 seasons.

\begin{tabular}{|c|c|c|c|c|c|c|c|c|c|c|c|c|}
\hline \multirow{2}{*}{ Treatments } & \multicolumn{3}{|c|}{ Pulp weight (gms) } & \multicolumn{3}{|c|}{ Seed weight (gms) } & \multicolumn{3}{|c|}{ Peel weight (gms) } & \multicolumn{3}{|c|}{ Seed/pulp ratio } \\
\hline & $2008 / 2009$ & $2009 / 2010$ & $2010 / 2011$ & $2008 / 2009$ & $2009 / 2010$ & 2010/2011 & 2008/2009 & $2009 / 2010$ & $2010 / 2011$ & $2008 / 2009$ & $2009 / 2010$ & $2010 / 2011$ \\
\hline $\begin{array}{l}\text { 1-Mineral nitrogen "MN" } \\
100 \% \text { (control) }\end{array}$ & $306.7^{\mathrm{g}}$ & $315.3^{\mathrm{f}}$ & $312.6^{\mathrm{g}}$ & $65.43^{\mathrm{a}}$ & $67.06^{\mathrm{a}}$ & $65.13^{\mathrm{a}}$ & $46.86^{\mathrm{f}}$ & $39.51^{\mathrm{e}}$ & $41.36^{\mathrm{e}}$ & $0.213^{\mathrm{a}}$ & $0.212^{\mathrm{a}}$ & $0.208^{\mathrm{a}}$ \\
\hline $\begin{array}{l}\text { 2- Mineral nitrogen "MN" } \\
80 \%+\text { Town refuse "TR" } \\
20 \%\end{array}$ & $330.8^{\mathrm{e}}$ & $341.8^{\mathrm{d}}$ & $337.4^{\mathrm{e}}$ & $54.00^{\mathrm{c}}$ & $58.10^{\mathrm{c}}$ & $53.90^{\mathrm{c}}$ & $62.86^{\mathrm{d}}$ & $53.83^{\mathrm{c}}$ & $56.55^{\mathrm{c}}$ & $0.163^{\mathrm{c}}$ & $0.170^{\mathrm{c}}$ & $0.759^{c}$ \\
\hline $\begin{array}{l}\text { 3- Mineral nitrogen "MN" } \\
50 \%+\text { Town refuse "TR" } \\
50 \%\end{array}$ & $356.2^{\mathrm{b}}$ & $368.7^{\mathrm{a}}$ & $363.8^{b}$ & $49.33^{\mathrm{e}}$ & $51.50^{f}$ & $49.20^{\mathrm{e}}$ & $69.50^{\mathrm{a}}$ & $63.46^{\mathrm{a}}$ & $60.90^{\mathrm{a}}$ & $0.138^{f}$ & $0.139^{f}$ & $0.135^{\mathrm{f}}$ \\
\hline $\begin{array}{l}\text { 4- Mineral nitrogen "MN" } \\
20 \%+\text { Town refuse "TR" } \\
80 \%\end{array}$ & $334.3^{\mathrm{d}}$ & $345.1^{\mathrm{c}}$ & $341.2^{\mathrm{d}}$ & $52.73^{\mathrm{d}}$ & $57.56^{\mathrm{d}}$ & $52.83^{\mathrm{d}}$ & $64.66^{\mathrm{c}}$ & $54.23^{\mathrm{c}}$ & $58.43^{\mathrm{b}}$ & $0.158^{\mathrm{d}}$ & $0.166^{\mathrm{d}}$ & $0.154^{\mathrm{d}}$ \\
\hline $\begin{array}{l}\text { 5- Mineral nitrogen "MN" } \\
80 \%+\text { Cattle manure } \\
\text { "CM" 20\% }\end{array}$ & $346.8^{\mathrm{c}}$ & $357.6^{\mathrm{b}}$ & $353.7^{\mathrm{c}}$ & $52.62^{\mathrm{d}}$ & $54.73^{\mathrm{e}}$ & $52.60^{\mathrm{d}}$ & $67.03^{b}$ & $58.20^{\mathrm{b}}$ & $60.40^{\mathrm{a}}$ & $0.152^{\mathrm{e}}$ & $0.153^{\mathrm{e}}$ & $0.149^{\mathrm{e}}$ \\
\hline $\begin{array}{l}\text { 6- Mineral nitrogen "MN" } \\
50 \%+\text { Cattle manure } \\
\text { "CM" 50\% }\end{array}$ & $318.2^{f}$ & $328.0^{\mathrm{e}}$ & $324.3^{f}$ & $57.60^{\mathrm{b}}$ & $59.40^{\mathrm{b}}$ & $57.16^{\mathrm{b}}$ & $56.76^{\mathrm{e}}$ & $49.40^{\mathrm{d}}$ & $51.16^{\mathrm{d}}$ & $0.181^{b}$ & $0.181^{b}$ & $0.176^{\mathrm{b}}$ \\
\hline $\begin{array}{l}\text { 7- Mineral nitrogen "MN" } \\
20 \%+\text { Cattle manure } \\
\text { "CM" 80\% }\end{array}$ & $358.8^{\mathrm{a}}$ & $370.1^{\mathrm{a}}$ & $368.0^{\mathrm{a}}$ & $49.23^{\mathrm{e}}$ & $49.73 \mathrm{~g}$ & $49.00^{\mathrm{e}}$ & $69.76^{\mathrm{a}}$ & $65.10^{\mathrm{a}}$ & $60.91^{\mathrm{a}}$ & $0.137^{\mathrm{g}}$ & $0.134^{\mathrm{g}}$ & $0.133^{\mathrm{g}}$ \\
\hline New L.S.D. at $5 \%$ & 1.6 & 2.2 & 1.5 & 0.42 & 0.38 & 0.50 & 1.19 & 2.56 & 1.88 & 0.001 & 0.002 & 0.002 \\
\hline
\end{tabular}

The values per column followed by the same letter do not differ at $5 \%$ level of significance.

$\mathrm{MN}=$ Mineral nitrogen $; \mathrm{TR}=$ Town refuse $; \mathrm{CM}=$ Cattle manure. 
Table (5): Effect of different organic nitrogen sources on chemical characteristics of Zebda mango fruits in 2008/2009, 2009/2010 and 2010/2011 seasons.

\begin{tabular}{|c|c|c|c|c|c|c|c|c|c|}
\hline \multirow{2}{*}{ Treatments } & \multicolumn{3}{|c|}{ TSS \% } & \multicolumn{3}{|c|}{ Acidity } & \multicolumn{3}{|c|}{ TSS/acid ratio } \\
\hline & $2008 / 2009$ & $2009 / 2010$ & $2010 / 2011$ & $2008 / 2009$ & $2009 / 2010$ & $2010 / 2011$ & $2008 / 2009$ & $2009 / 2010$ & $2010 / 2011$ \\
\hline 1-Mineral nitrogen "MN" 100\% (control) & $12.56^{\mathrm{g}}$ & $14.33^{\mathrm{f}}$ & $13.45^{\mathrm{g}}$ & $0.380^{\mathrm{a}}$ & $0.348^{\mathrm{a}}$ & $0.372^{\mathrm{a}}$ & $33.05^{\mathrm{g}}$ & $41.17^{\mathrm{g}}$ & $36.15^{\mathrm{f}}$ \\
\hline 2- Mineral nitrogen "MN" 80\%+ Town refuse "TR" 20\% & $13.16^{\mathrm{e}}$ & $15.10^{\mathrm{d}}$ & $14.06^{\mathrm{e}}$ & $0.300^{\mathrm{c}}$ & $0.273^{\mathrm{bc}}$ & $0.293^{\mathrm{bc}}$ & $43.86^{\mathrm{e}}$ & $55.31^{\mathrm{e}}$ & $47.98^{\mathrm{d}}$ \\
\hline 3- Mineral nitrogen "MN" 50\%+ Town refuse "TR" 50\% & $14.43^{b}$ & $16.26^{\mathrm{b}}$ & $15.44^{b}$ & $0.220^{\mathrm{e}}$ & $0.213^{\mathrm{d}}$ & $0.215^{\mathrm{d}}$ & $65.59^{b}$ & $76.33^{b}$ & $71.81^{b}$ \\
\hline 4- Mineral nitrogen "MN" 20\%+ Town refuse "TR" 80\% & $13.26^{\mathrm{d}}$ & $15.16^{\mathrm{d}}$ & $14.17^{\mathrm{d}}$ & $0.296^{\mathrm{c}}$ & $0.272^{b c}$ & $0.289^{b c}$ & $44.19^{d}$ & $55.73^{\mathrm{e}}$ & $49.03^{d}$ \\
\hline 5- Mineral nitrogen "MN" 80\%+ Cattle manure "CM" 20\% & $13.90^{\mathrm{c}}$ & $15.56^{\mathrm{c}}$ & $14.93^{c}$ & $0.259^{\mathrm{e}}$ & $0.238^{\mathrm{cd}}$ & $0.253^{\mathrm{cd}}$ & $53.66^{\mathrm{c}}$ & $65.37^{\mathrm{c}}$ & $59.01^{\mathrm{c}}$ \\
\hline 6- Mineral nitrogen "MN" 50\%+ Cattle manure "CM" 50\% & $12.90^{\mathrm{f}}$ & $14.70^{\mathrm{e}}$ & $13.89^{f}$ & $0.337^{b}$ & $0.308^{\mathrm{b}}$ & $0.328^{\mathrm{b}}$ & $38.27^{\mathrm{f}}$ & $47.72^{\mathrm{f}}$ & $42.34^{\mathrm{e}}$ \\
\hline 7- Mineral nitrogen "MN" 20\%+ Cattle manure "CM" 80\% & $14.50^{\mathrm{a}}$ & $16.40^{\mathrm{a}}$ & $15.56^{\mathrm{a}}$ & $0.217^{\mathrm{e}}$ & $0.211^{\mathrm{d}}$ & $0.212^{\mathrm{d}}$ & $66.82^{\mathrm{a}}$ & $77.72^{\mathrm{a}}$ & $73.39^{\mathrm{a}}$ \\
\hline New L.S.D. at $5 \%$ & 0.06 & 0.09 & 0.05 & 0.035 & 0.040 & 0.043 & 0.82 & 1.04 & 1.91 \\
\hline
\end{tabular}

Cont. Table (5): Effect of different organic nitrogen sources on chemical characteristics of Zebda mango fruits in 2008/2009, 2009/2010 and 2010/2011 seasons.

\begin{tabular}{|c|c|c|c|c|c|c|c|c|c|}
\hline \multirow{2}{*}{ Treatments } & \multicolumn{3}{|c|}{ Reducing sugars \% } & \multicolumn{3}{|c|}{ Total sugars $\%$} & \multicolumn{3}{|c|}{ Carotinoids mg/100 gm } \\
\hline & $2008 / 2009$ & $2009 / 2010$ & $2010 / 2011$ & $2008 / 2009$ & $2009 / 2010$ & $2010 / 2011$ & $2008 / 2009$ & $2009 / 2010$ & $2010 / 2011$ \\
\hline 1-Mineral nitrogen "MN" 100\% (control) & $4.896^{\mathrm{f}}$ & $3.660^{\mathrm{f}}$ & $4.800^{\mathrm{f}}$ & $12.874^{\mathrm{g}}$ & $12.450^{\mathrm{g}}$ & $12.746^{\mathrm{g}}$ & $1.908^{\mathrm{g}}$ & $1.935^{\mathrm{g}}$ & $1.923 \mathrm{~g}$ \\
\hline 2- Mineral nitrogen "MN" 80\%+ Town refuse "TR" $20 \%$ & $6.043^{\mathrm{d}}$ & $4.610^{\mathrm{d}}$ & $6.180^{\mathrm{d}}$ & $13.110^{\mathrm{e}}$ & $13.433^{\mathrm{e}}$ & $13.943^{\mathrm{e}}$ & $2.076^{\mathrm{e}}$ & $2.050^{\mathrm{e}}$ & $2.093^{\mathrm{e}}$ \\
\hline 3- Mineral nitrogen "MN" 50\%+ Town refuse "TR" 50\% & $6.850^{\mathrm{a}}$ & $5.126^{\mathrm{a}}$ & $7.000^{\mathrm{a}}$ & $14.020^{\mathrm{b}}$ & $14.196^{\mathrm{b}}$ & $14.660^{\mathrm{b}}$ & $2.226^{\mathrm{b}}$ & $2.200^{\mathrm{b}}$ & $2.247^{\mathrm{b}}$ \\
\hline 4- Mineral nitrogen "MN" 20\%+ Town refuse "TR" $80 \%$ & $6.133^{\mathrm{c}}$ & $4.693^{\mathrm{c}}$ & $6.270^{\mathrm{c}}$ & $13.330^{\mathrm{d}}$ & $13.540^{\mathrm{d}}$ & $14.060^{\mathrm{d}}$ & $2.083^{\mathrm{d}}$ & $2.066^{\mathrm{d}}$ & $2.105^{\mathrm{d}}$ \\
\hline 5- Mineral nitrogen "MN" 80\%+ Cattle manure "CM" 20\% & $6.496^{\mathrm{b}}$ & $4.920^{\mathrm{b}}$ & $6.643^{b}$ & $13.416^{\mathrm{c}}$ & $13.870^{\mathrm{c}}$ & $14.420^{\mathrm{c}}$ & $2.156^{\mathrm{c}}$ & $2.130^{\mathrm{c}}$ & $2.173^{\mathrm{c}}$ \\
\hline 6- Mineral nitrogen "MN" 50\%+ Cattle manure "CM" 50\% & $5.696^{\mathrm{e}}$ & $3.990^{\mathrm{e}}$ & $5.683^{\mathrm{e}}$ & $13.221^{\mathrm{f}}$ & $13.213^{\mathrm{f}}$ & $13.573^{f}$ & $2.005^{\mathrm{f}}$ & $1.990^{\mathrm{f}}$ & $2.020^{\mathrm{f}}$ \\
\hline 7- Mineral nitrogen "MN" 20\%+ Cattle manure "CM" $80 \%$ & $6.866^{\mathrm{a}}$ & $5.160^{\mathrm{a}}$ & $7.020^{\mathrm{a}}$ & $14.426^{\mathrm{a}}$ & $14.303^{\mathrm{a}}$ & $14.780^{\mathrm{a}}$ & $2.236^{\mathrm{a}}$ & $2.223^{\mathrm{a}}$ & $2.253^{\mathrm{a}}$ \\
\hline New L.S.D. at $5 \%$ & 0.069 & 0.051 & 0.033 & 0.012 & 0.009 & 0.015 & 0.006 & 0.008 & 0.004 \\
\hline
\end{tabular}

The values per column followed by the same letter do not differ at $5 \%$ level of significance.

$\mathrm{MN}=$ Mineral nitrogen ; $\mathrm{TR}=$ Town refuse $; \mathrm{CM}=$ Cattle manure 
Table (6): Effect of different organic nitrogen sources on leaf mineral content of Zebda mango trees in 2008/2009, 2009/2010 and 2010/2011 seasons.

\begin{tabular}{|c|c|c|c|c|c|c|c|c|c|}
\hline \multirow{2}{*}{ Treatments } & \multicolumn{3}{|c|}{ Nitrogen \% } & \multicolumn{3}{|c|}{ Phosphorus \% } & \multicolumn{3}{|c|}{ Potassium \% } \\
\hline & 2008/2009 & $2009 / 2010$ & 2010/2011 & $2008 / 2009$ & $2009 / 2010$ & 2010/2011 & $2008 / 2009$ & $2009 / 2010$ & $2010 / 2011$ \\
\hline 1-Mineral nitrogen "MN" 100\% (control) & $0.840^{\mathrm{g}}$ & $0.798^{\mathrm{g}}$ & $0.849 \mathrm{~g}$ & $0.159^{\mathrm{c}}$ & $0.117^{\mathrm{d}}$ & $0.175^{\mathrm{c}}$ & $0.870^{\mathrm{c}}$ & $0.849^{\mathrm{b}}$ & $0.853^{\mathrm{b}}$ \\
\hline 2- Mineral nitrogen "MN" 80\%+ Town refuse "TR" 20\% & $1.523^{\mathrm{e}}$ & $1.459^{\mathrm{e}}$ & $1.535^{\mathrm{e}}$ & $0.198^{\mathrm{b}}$ & $0.163^{b}$ & $0.217^{\mathrm{b}}$ & $1.123^{\mathrm{bc}}$ & $1.194^{\mathrm{ab}}$ & $1.160^{\mathrm{ab}}$ \\
\hline 3- Mineral nitrogen "MN" 50\%+ Town refuse "TR" 50\% & $1.896^{\mathrm{b}}$ & $1.773^{\mathrm{b}}$ & $1.914^{\mathrm{b}}$ & $0.235^{\mathrm{a}}$ & $0.206^{\mathrm{a}}$ & $0.244^{\mathrm{a}}$ & $1.283^{\mathrm{ab}}$ & $1.236^{\mathrm{a}}$ & $1.324^{\mathrm{a}}$ \\
\hline 4- Mineral nitrogen "MN" 20\%+ Town refuse "TR" 80\% & $1.560^{\mathrm{d}}$ & $1.470^{\mathrm{d}}$ & $1.573^{\mathrm{d}}$ & $0.224^{\mathrm{a}}$ & $0.185^{\mathrm{a}}$ & $0.203^{\mathrm{b}}$ & $1.233^{\mathrm{abc}}$ & $1.197^{\mathrm{ab}}$ & $1.283^{\mathrm{a}}$ \\
\hline 5- Mineral nitrogen "MN" 80\%+ Cattle manure "CM" 20\% & $1.723^{\mathrm{c}}$ & $1.615^{\mathrm{c}}$ & $1.736^{\mathrm{c}}$ & $0.225^{\mathrm{a}}$ & $0.203^{\mathrm{a}}$ & $0.226^{\mathrm{a}}$ & $1.236^{\mathrm{abc}}$ & $1.210^{\mathrm{ab}}$ & $1.303^{\mathrm{a}}$ \\
\hline 6- Mineral nitrogen "MN" 50\%+ Cattle manure "CM" 50\% & $1.320^{\mathrm{f}}$ & $1.267^{\mathrm{f}}$ & $1.330^{\mathrm{f}}$ & $0.177^{\mathrm{c}}$ & $0.139^{\mathrm{c}}$ & $0.180^{\mathrm{c}}$ & $1.233^{\mathrm{bc}}$ & $1.055^{\mathrm{ab}}$ & $1.082^{\mathrm{ab}}$ \\
\hline 7- Mineral nitrogen "MN" 20\%+ Cattle manure "CM" 80\% & $1.920^{\mathrm{a}}$ & $1.806^{\mathrm{a}}$ & $1.935^{\mathrm{a}}$ & $0.237^{\mathrm{a}}$ & $0.218^{\mathrm{a}}$ & $0.246^{\mathrm{a}}$ & $1.515^{\mathrm{a}}$ & $1.413^{\mathrm{a}}$ & $1.335^{\mathrm{a}}$ \\
\hline New L.S.D. at $5 \%$ & 0.007 & 0.008 & 0.01 & 0.023 & 0.020 & 0.022 & 0.390 & 0.384 & 0.367 \\
\hline
\end{tabular}

Cont. Table (6): Effect of different organic nitrogen sources on leaf mineral content of Zebda mango trees in 2008/2009, 2009/2010 and 2010/2011 seasons.

\begin{tabular}{|c|c|c|c|c|c|c|c|c|c|}
\hline \multirow{2}{*}{ Treatments } & \multicolumn{3}{|c|}{ Iron (ppm) } & \multicolumn{3}{|c|}{ Manganese (ppm) } & \multicolumn{3}{|c|}{ Zinc (ppm) } \\
\hline & $2008 / 2009$ & $2009 / 2010$ & 2010/2011 & $2008 / 2009$ & $2009 / 2010$ & 2010/2011 & $2008 / 2009$ & $2009 / 2010$ & 2010/2011 \\
\hline 1-Mineral nitrogen "MN" 100\% (control) & $55.3^{\mathrm{g}}$ & $57.6^{\mathrm{f}}$ & $56.6^{\mathrm{e}}$ & $41.4^{\mathrm{f}}$ & $40.3^{\mathrm{f}}$ & $43.0^{\mathrm{f}}$ & $47.5^{\mathrm{g}}$ & $58.0^{\mathrm{g}}$ & $50.6^{\mathrm{g}}$ \\
\hline 2- Mineral nitrogen "MN" 80\%+ Town refuse "TR" 20\% & $67.2^{\mathrm{e}}$ & $69.0^{\mathrm{d}}$ & $68.8^{\mathrm{c}}$ & $46.3^{\mathrm{d}}$ & $45.8^{\mathrm{e}}$ & $48.1^{\mathrm{d}}$ & $51.8^{\mathrm{d}}$ & $67.4^{\mathrm{e}}$ & $55.0^{\mathrm{e}}$ \\
\hline 3- Mineral nitrogen "MN" 50\%+ Town refuse "TR" 50\% & $80.3^{\mathrm{b}}$ & $81.9^{\mathrm{a}}$ & $82.2^{\mathrm{a}}$ & $57.4^{\mathrm{b}}$ & $70.2^{\mathrm{b}}$ & $59.4^{\mathrm{b}}$ & $64.1^{\mathrm{b}}$ & $71.8^{\mathrm{b}}$ & $68.0^{\mathrm{b}}$ \\
\hline 4- Mineral nitrogen "MN" 20\%+ Town refuse "TR" $80 \%$ & $69.5^{\mathrm{d}}$ & $70.0^{\mathrm{c}}$ & $71.3^{\mathrm{b}}$ & $53.4^{\mathrm{c}}$ & $56.1^{\mathrm{d}}$ & $55.7^{\mathrm{c}}$ & $58.9^{\mathrm{c}}$ & $70.6^{\mathrm{d}}$ & $63.1^{\mathrm{d}}$ \\
\hline 5- Mineral nitrogen "MN" 80\%+ Cattle manure "CM" 20\% & $70.1^{\mathrm{c}}$ & $72.0^{\mathrm{b}}$ & $71.8^{\mathrm{b}}$ & $57.0^{\mathrm{b}}$ & $59.6^{\mathrm{c}}$ & $59.2^{\mathrm{b}}$ & $60.0^{\mathrm{b}}$ & $71.2^{\mathrm{c}}$ & $63.7^{\mathrm{c}}$ \\
\hline 6- Mineral nitrogen "MN" 50\%+ Cattle manure "CM" 50\% & $61.0^{f}$ & $64.8^{\mathrm{e}}$ & $62.4^{\mathrm{d}}$ & $42.7^{\mathrm{e}}$ & $45.5^{\mathrm{e}}$ & $44.3^{\mathrm{e}}$ & $51.3^{\mathrm{e}}$ & $61.7^{\mathrm{f}}$ & $54.4^{\mathrm{f}}$ \\
\hline 7- Mineral nitrogen "MN" 20\%+ Cattle manure "CM" 80\% & $80.9^{\mathrm{a}}$ & $82.4^{\mathrm{a}}$ & $82.8^{\mathrm{a}}$ & $64.1^{\mathrm{a}}$ & $73.6^{\mathrm{a}}$ & $66.6^{\mathrm{a}}$ & $64.6^{\mathrm{a}}$ & $72.5^{\mathrm{a}}$ & $68.6^{\mathrm{a}}$ \\
\hline New L.S.D. at $5 \%$ & 0.5 & 0.6 & 0.6 & 0.7 & 0.4 & 0.3 & 0.5 & 0.2 & 0.3 \\
\hline
\end{tabular}

The values per column followed by the same letter do not differ at $5 \%$ level of significance. $\mathrm{MN}=$ Mineral nitrogen ; TR $=$ Town refuse ; CM =Cattle manure. 
nitrogen source $(\mathrm{MN})$ significantly improved leaf mineral contents compared with the application of nitrogen mineral source only. Treatment of (MN $20 \%+\mathrm{CM} 80 \%$ ) significantly increased nitrogen, manganese and zinc leaf contents more than those of the other treatments and the control. At the sametime, either treatments, (MN 20\% + CM $80 \%$ ) or ( MN $50 \%+$ TR $50 \%$ ) significantly increased phosphorus, potassium and iron leaf contents than those of the remained treatments in most cases.

On the other hand, the least leaf mineral contents were recorded for treatment of (MN $100 \%$, control). These results were true in the three experimental seasons.

The beneficial effect of the organic $\mathrm{N}$ sources on reducing soil $\mathrm{pH}$ may be responsible for facilitating the availability of most nutrients and this could be explained by the present results. In addition, the studied organic $\mathrm{N}$ source (cattle manure and town refuse) contained high amounts of other nutrients (Table 2). Also, the increase in leaf mineral contents may be explained on the basis that the organic materials increased the soil water holding capacity which encourage the solubility and consequently the available nutrients (Zaid \& Kariem, 1992; El-Kassas et al., 1997 and Nassar, 1998). When organic and conventional chemical fertilizer $\mathrm{N}$ were applied together, nutrients uptake was higher than with organic $\mathrm{N}$ or mineral $\mathrm{N}$ alone as shown in Table (6). This may be explained on the basis that the combined addition of organic and inorganic $\mathrm{N}$ maintains continuous satisfactory increase of the efficiency of nutrient utilization and reduced nutrient losses (Harhash \& Abdel-Nasser,2000). These results are in agreement with those obtained by El-Sayed (1994); Ahmed et al. (1996); Mansour \& Ahmed (1998); Hammam et al. (2003); Saad \& Saad (2007) and Al-Wasfy \& El-Khawaga (2008).

In general, the improvement in yield and fruit quality of Zebda mango trees due to the application of organic manures were reflecting soil properties i.e., soil aggregation, soil water retention and lowering $\mathrm{pH}$ which leads to volatilization of nutrients and increase their availability and supply to trees (Gouda, 1984; Salem, 1986 and Dahdoh \& El-Hassanin, 1993) also, organic matter as complexing agent, minimizes the loss of nutrients by leaching (Abdel-Nasser and Harhash,2000). In meantime, using organic manures as a fraction of $\mathrm{N}$ requirements reduced the fertilization costs and decreasing the losses in $\mathrm{NO}_{3}^{-}$that causes the groundwater pollution which caused by excess of chemical fertilizers (Cole et al., 1987).

Finally, it can be recommended from the present data that partially replacing of mineral $\mathrm{N}$ fertilizer requirement by organic manures such as cattle manure or town refuse was useful, whereas annual application of mineral nitrogen $20 \%$ plus cattle manure $80 \%$ or mineral nitrogen $50 \%$ plus town refuse $50 \%$ per tree produced the highest yield with fairly good quality of Zebda mango fruits.

\section{REFERENCES}

Abd El-Hameed M.A. and Ragab M.A. (2004). Response of Sewy date palm to application of some organic fertilizers. Abstract of the Second Conf. on Date Palm. Fac. Agric., El-Arish, Suez Canal Univ., Egypt.

Abd El-Naby S.K.M. and Gomaa A.M. (2000). Growth, nutrition status, yield and fruit quality of Maghrabi banana as affected by some organic manures and biofertilizer. Minufiya J. Agric. Res., 25 (4): 1113-1129.

Abd El-Nasser G.and Harhash M.M (2000). Effect of organic manures in combination with elemental sulphur on soil physical and chemical characteristics, yield, fruit quality, leaf water contents and nutritional status of Flame seedless grapevines. I- Soil physical and chemical characteristics. J. of Agric. Sci., Mansoura Univ., 25 (6): 3541-3558.

Abd El-Rahman A.E. (2007). Evaluation of organic fertilization in Thompson seedless vineyards. M.Sc.Thesis, Fac. Agric., Mansoura Univ., Egypt.

Abd El-Salam, Yasmin G., Nomeir Safaa A. and Al-Ashkar R.A. (2009). Using of some bio and organic fertilizers to reduce the rate of mineral $\mathrm{N}$ fertilization and improving orange tree production. Zagzig J. Agric. Res., Vol.36 No.(4): 691-719.

Ahmed F.F., Ragab M.A. and Mansour A.E.M. (1996). A comparative study on the effects of farmyard manure and filter mud for Red Roomy grapevines (Visits vinifers L.). $1^{\text {st }}$ Egypt-Hung. Nort. Conf., 2, 232.

Al-Wasfy M.M. and El-Khawaga A.A.S. (2008). Effect of organic fertilization on growth, yield and fruit quality of Zaghloul date palm growth in sandy soil. Assiut J. of Agric. Sci., 39 (1): 121-133.

A.O.A.C. (1995). Association of Official Agricultural Chemists, Official Methods of Analysis $15^{\text {th }}$ Ed. Published by A.O.A.C. 
Washington, D.C. (USA).

Casale W.L., Minassian V., Menge J.A., Lovatt C.J., P and E., Johnason E. and Guillement F. (1995). Urban agricultural wasts for use as delivery of microbial biocontrol agents. J. Hort. Sci. 70 (2): 315-332.

Chatzitheodorou I.T., Sotiropoulos T.E. and Mouhtaridou G.I. (2004). Effect of nitrogen phosphorus, potassium fertilization and manure on fruit yield and fruit quality of the peach cultivars "Spring Time" and "Red Hayen". Agronomy Research, 2(2): 135143.

Cole C.V., Williams J., Shaffer M. and Hanson J. (1987). Nutrient and organic matter as components of agricultural production system models. Pp. 147-166. In "Soil fertility and organic matter component of production systems", Follett et al. (eds)SSSA, Spec. Publ. No.19 Madison, WI.

Dahdoh M.S.A. and El-Hassanin A.S. (1993). Combined effects of organic sourcse, irrigation water salinity and moisture level on the growth and mineral composition of barley grown on calcareous soil. Desert Inst. Bull., 43, Suppl., 123-138.

Diab Y.M. (2006). Effect of some cultural practices on yield and fruit quality of Phoenix dactylifera L. cv. Sewy under New Valley conditions. M.Sc. Thesis; Fac. Agric., Assiut Univ., Egypt.

El-Assar A.M. (2005). Response of "Zaghloul" date yield and fruit characteristics to various organic and inorganic fertilization types as well as fruit thinning models in a rich carbonate soil. J. Agric. Sci., Mansoura Univ., 30 (5): 2795-2814.

El-Kassas H.I., Abou-Hadid A.F. and Eissa N.M.H. (1997). Effect of different organic manures on the yield and elemental composition of sweet pepper plants grown on sandy soil. Egypt. J. Sci. 12 (3): 262281.

El-Sayed M.A. (1994). The benefits of some organic nitrogen fertilizers on Red Roomy grapevines (Vitis vinifera L.). Minia J. Agric. Res. \& Dev.16 No.3, 1295.

Engelstad O.P. and Russel S.A. (1975). Fertilizers for use under tropical conditions. Adv. Agron. 27: 175-208.

Evenhuis B. (1976). Nitrogen determination. Dept. Agric. Res. Royal Tropical Instit., Amsterdam.

Gambrell R.P., Gilliam J.W. and Week S.B. (1975). Nitrogen losses from soils of the
North Carolina coastal plain. J. Environ. Qual. 4: 317-322.

Geetha K. and Nair R.R. (2000). Integrated plant nutrition system (IPNS) for banana. Ann. of Agric. Res., (India) 21 (4): 499-503.

Goring C.A.L. (1962). Control nitrification by 2chloro-6-(trichloro-methyl) pyrine. Soil Sci. 93: 211-218.

Gouda M.A.K. (1984). Soil and water management of sandy soil Ph.D. Thesis, Fac. of Agric., Zagazig Univ., Egypt.

Hammam M.S., Ibrahim E.G. and Mansour A.E.M. (2003). Response of Williams banana to some organic nitrogen fertilizers. Egypt. J. Hort. 30, No.1-2, pp.51-65.

Harhash M.M. and Abd El-Nasser G. (2000). Effect of organic manures in combination with elemental sulphur on soil physical and chemical characteristics, yield, fruit quality, leaf water contents and nutritional status of Flame seedless grapevines II yield, fruit quality, leaf water contents and nutritional status. J. Agric. Sci., Mansoura Univ., 25 (5): 2819-2837.

Hossam El-Deen A.S. and Boshra E.S. (2008). Effect of different sources of organic fertilizers as a partial substitute for mineral nitrogen fertilizer of Williams banana. J. Agric. Sci., Mansoura Univ., 33 (6): 43694381.

Joo Y.H. Lee, Senanayake Y.I.A. and Sangakkara I.R. (1999). Effect of EM on the production of crops and waste treatment in Korea. Fifth International Conference on Kyusei Nature Farming, Bangkok, Thialand, 23-26 October, 1997, 151-156.

Li X.J., Dona S.F. and Liu Y.S. (1998). Determination of IAA and cytokinins in the soil with different organic manure for pot cultured apple. Plant Physiology Communications, 34 (3): 183-185.

Malik C.P. and Singh M.B. (1980). Plant enzymology and histoenzymology. A Text Manual, pp.276-277, Kalyani publishers, New Delhi.

Mansour A.E.M. and Ahmed F.F. (1998). Productivity of Bisinnara mangoes as infuenced with the application of filter mud and farmyard manure. Egypt. J. Hort. 25, 2, 179.

Mansour A.E.M., Ahmed F.F. and Ahmed Y.M. (2004). Effect of bio. and organic sources of $\mathrm{N}$ as a partial substitute for mineral fertilizer on fruiting of Sewy date palms. The Second International Conference on 
Date Palm, Fac. Agric., El-Arish, Suez Canal Univ.

Mead R., Curnow R.N. and Harted A.M. (1993). Statistical Methods in Agricultural and Experimental Biology. $2^{\text {nd }}$ Ed. Chapman \& Hall, London.

Ministry of Agriculture \& Land Reclamation, Economic Affairs Sector, Egypt (2008). Study of important indicators of the agricultural statistics. Vol.2: P.331.

Mohamed G.A. and Gobara A.A. (2004). Response of Sewy date palms grown under New Valley conditions to organic, bio and mineral fertilization. Minia J. of Agric. Res. \& Develop., 24 (3): 397-414.

Murphy J. and Riley J.P. (1962). A modified single solution method for the determination of phosphorus in natural waters. Anal. Chem. Acta. 27: 31-36.

Nassar I.N. (1998). Utilization of the municipal garbage (MG) as a soil amendment. Alex. J. Agric. Res., 43 (3): 317-332.

Omar A.E. and Belal E.B. (2007). Effect of organic, inorganic and bio-fertilizer application on fruit yield and quality of mango trees (Magnifera indica L. cv. "Sukari" in Balteem, Kafer El-Sheikh, Egypt. J. Agric. Res., Kafer El-Sheikh Univ., 33 (4): 857-872.

Roy S.K. (1973). Simple and rapid method for estimation of total carotenoid pigments in mango. J. Food Science and Technology, 10: P.45.

Saad R.I. and Saad M.M. (2007). Effect of different organic nitrogen sources on growth, yield and fruit quality of Williams banana. J. Adv. Agric. Res., Vol.12 (1), 149-164.
Sahrawat K.L. (1979). Nitrogen losses in rice soils. Fert. News 24: 38-48.

Salem N.M.M. (1986). Agrochemical aspects related to the use of conditioners and organic wastes in soils. Ph.D. Thesis, Fac. Agric. Sci. Rijks Univ. Gent, Belgium.

Shaheen M.A., Eissa M.A., Saad M.M. and Mahmoud S.M. (2009). Influence of organic and biofertilization on growth, yield and fruit quality of Williams banana. J. Agric. Sci., Mansoura Univ., 34 (7): 80138025.

Shahein A.H., Attalla A.M., Kassem H.A. and Aly H.S.H. (2003). Effect of applying different organic and inorganic nitrogen sources to Zaghloul and Samany date palm cultivars on: II- Yield, fruit quality and fruit content of some pollutants. Proceedings of the International Conference on Date Palm. King Saud University. Kingdom of Saudi Arabia. P.195-205.

Smith B.L. (1998). Microorganisms in soil benefit growth and yield of banana. Netropika Bulletin, (299): 22-25. (C.F. Hort. Abstr., 68 (11): 10034).

Tiwary D.K., Hassan M.A. and Chattopadhyay P.K. (1999). Leaf nutrient and chlorophyll content in banana (Musa AAA) under the influence of Azotobacter and Azospirillum inoculation. Environment and Ecology, 17 (2): 346-350.

Zaid M.S. and Kriem H.M. (1992). Effect of sugar cane wastes and sulphur on some soil properties and nutrients uptake by Zea maize plants in sandy soil. J. Agric. Sci., Mansoura Univ., 17 (1): 181-188.

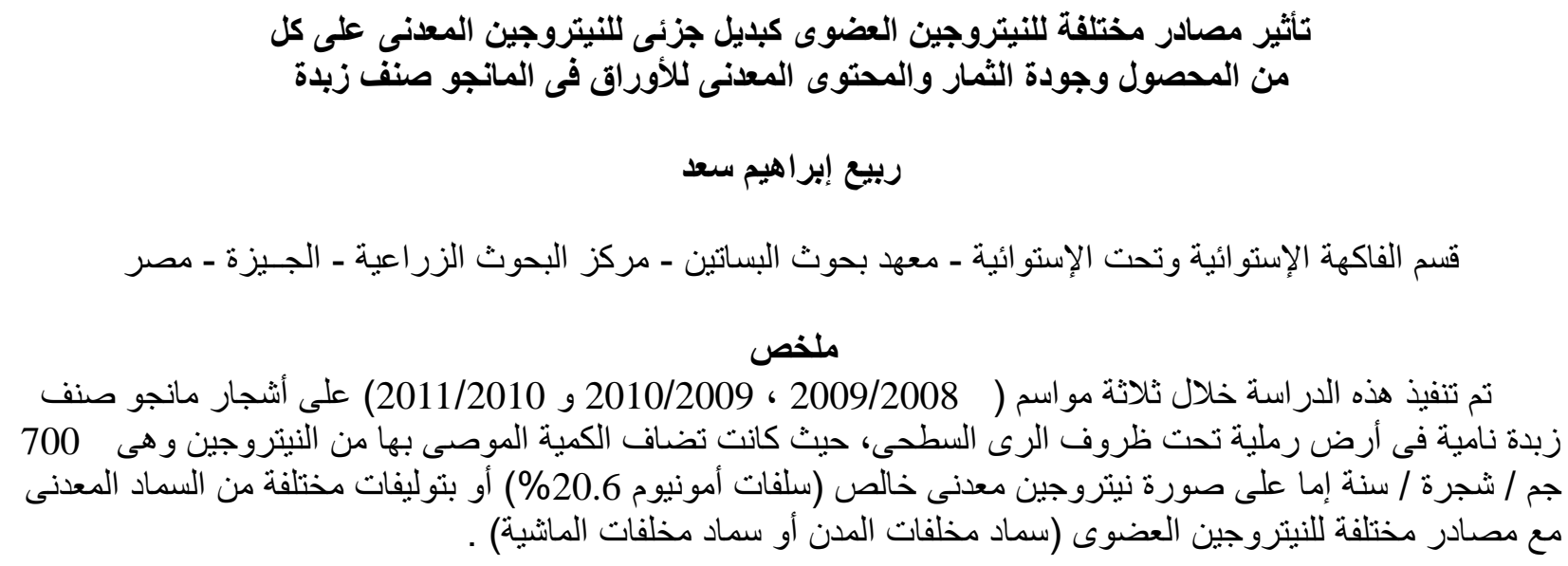




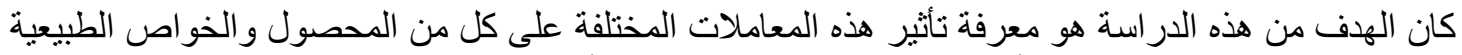

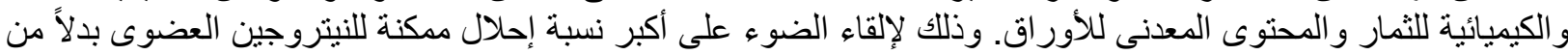

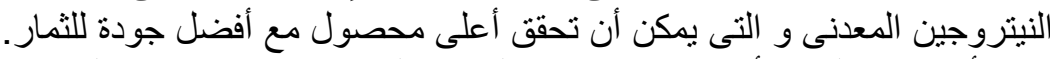

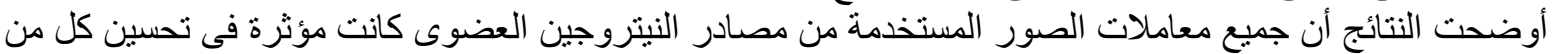

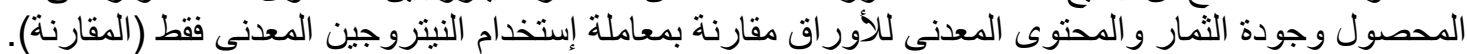

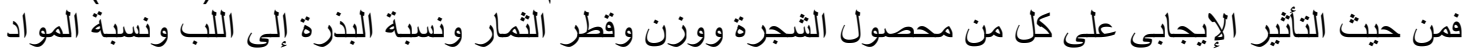

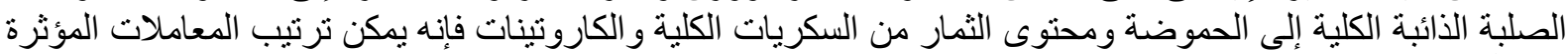

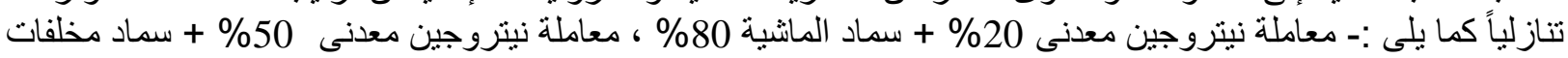

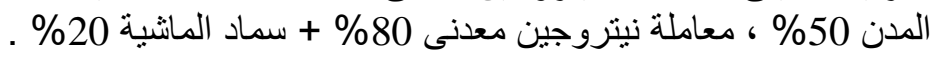

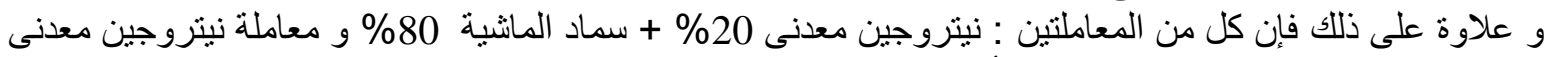

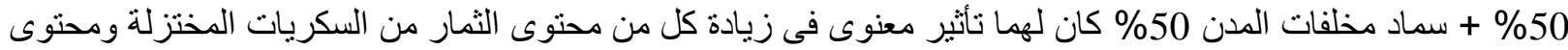

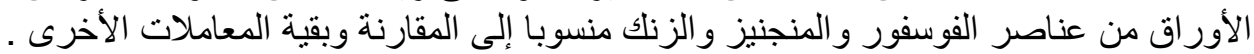

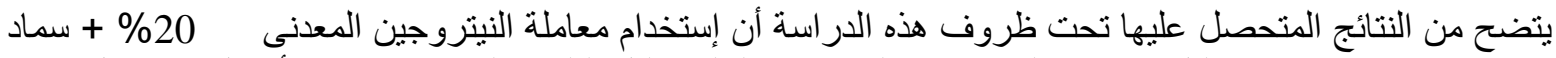

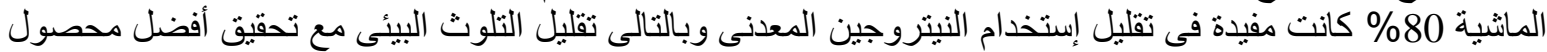

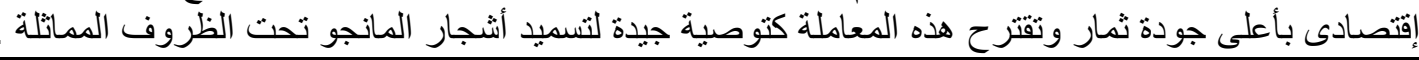

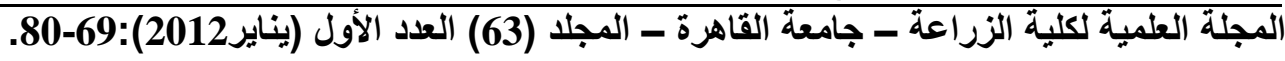

